\title{
Resultados imediatos da correção cirúrgica de escoliose idiopática do adolescente por via posterior com instrumentação após liberação anterior por videotoracoscopia
}

\author{
Short term results of surgical correction of adolescents' idiopathic \\ scoliosis by posterior approach instrumentation following \\ videothoracoscopic anterior release \\ Resultados inmediatos de la corrección quirúrgica de escoliosis \\ idiopática del adolescente por vía posterior con instrumentación \\ después de liberación anterior por videotoracoscopia
}

\author{
Eduardo Barros Puertas ${ }^{1}$ \\ David Del Curto² \\ Renato Hiroshi Salvioni Ueta ${ }^{2}$ \\ Délio Eulálio Martins Filho ${ }^{3}$ \\ Marcelo Wajchenberg ${ }^{3}$
}

\section{RESUMO}

Objetivo: descrever os resultados imediatos da aplicação da liberação anterior por videotoracoscopia, seguida de instrumentação por via posterior para correção cirúrgica da escoliose idiopática do adolescente. Métodos: foram selecionados 20 pacientes, no período de agosto de 1989 a maio de 2001, com escoliose idiopática do adolescente, padrão de curva torácica direita rígida, King III. Inicialmente realizou-se a abordagem pela via anterior com liberação por videotoracoscopia e depois a via posterior para correção e fixação com instrumental de Hartshill, em um mesmo tempo cirúrgico. Resultados: todos os procedimentos foram completados com sucesso com videotoracoscopia, sem necessidade de conversão para toracotomia. A média de idade

\begin{abstract}
Objective: to describe the initial results of anteriorvideothoracoscopic release followed by posterior instrumentation for surgical correction of adolescent idiopathic scoliosis. Methods: twenty patients were selected, between August 1989 and May 2001, with stiff, King III, right thoracic adolescent idiopathic scoliosis. They underwent anterior videothoracoscopic release followed by posterior correction and fixation with Hartshill instrumentation, performed in a single surgical stage. Results: all procedures were successfully completed with videothoracoscopy, without need to convert to thoracotomy. The average age was 15.7 years (12 to 21 years). Concerning the length
\end{abstract}

\section{RESUMEN}

Objetivo: describir los resultados inmediatos de la aplicación de la liberación anterior por videotoracoscopia, seguida de instrumentación por vía posterior para corrección quirúrgica de escoliosis idiopática del adolescente. Métodos: fueron seleccionados 20 pacientes, en el periodo de agosto de 1989 a mayo de 2001, con escoliosis idiopática del adolescente, con un padrón de curva torácica derecha rígida, King III. Inicialmente se realizó un abordaje por la vía anterior con liberación por videotoracoscopia y después la vía posterior para corrección y fijación con instrumental de Hartshill, en un mismo tiempo quirúrgico. Resultados: todos los procedimientos fueron completados con suceso con videotoracoscopia, sin necesidad de conversión para toraco-

\footnotetext{
Trabalho apresentado como parte da Tese de Livre Docência realizada na Escola Paulista de Medicina da Universidade Federal de São Paulo - UNIFESP - São Paulo (SP), Brasil (2001).

'Livre-docente, Professor-Associado da Disciplina de Ortopedia do Departamento de Ortopedia e Traumatologia da Escola Paulista de Medicina da Universidade Federal de São Paulo - UNIFESP - São Paulo (SP), Brasil.

${ }^{2}$ Pós-graduando (Mestrado) no Programa de Pós-graduação em Ortopedia e Traumatologia da Escola Paulista de Medicina da Universidade Federal de São Paulo - UNIFESP - São Paulo (SP), Brasil.

${ }^{3}$ Pós-graduando (Doutorado) no Programa de Pós-graduação em Ortopedia e Traumatologia da Escola Paulista de Medicina da Universidade Federal de São Paulo - UNIFESP - São Paulo (SP), Brasil.
} 
dos pacientes foi de 15,7 anos (12 a 21 anos). Em relação aos dias de internação, obteve-se um tempo médio de permanência de 9,45 dias ( 5 a 26 dias). A média entre os valores angulares foi, no pré-operatório, de $66,25^{\circ} \mathrm{Cobb}$ ( $48^{\circ}$ a $\left.92^{\circ} \mathrm{Cobb}\right)$, e no pós-operatório, de $31,2^{\circ} \mathrm{Cobb}\left(15^{\circ}\right.$ a $\left.45^{\circ} \mathrm{Cobb}\right)$. Como complicações, quatro pacientes tiveram nevralgia intercostal $(20 \%)$ e um caso de atelectasia (5\%). Conclusões: a discectomia por videotoracoscopia é um método efetivo e seguro para tornar flexíveis as curvas escolióticas rígidas. No entanto, trata-se de um procedimento tecnicamente difícil, que requer treinamento árduo e prolongado, com curva de aprendizado extensa. of hospital stay, there was a mean of 9.45 days (5 to 26 days). The preoperative $\mathrm{Cobb}$ angle average was $66.25^{\circ}\left(48^{\circ}\right.$ to $\left.92^{\circ}\right)$, and the mean postoperative Cobb angle was $31.2^{\circ}\left(15^{\circ}\right.$ to $\left.45^{\circ}\right)$. As complications, there were four patients with intercostal neuralgia (20\%) and one with atelectasis (5\%). Conclusions: the videothoracoscopic discectomy is a safe and effective method for making flexible the rigid scoliotic curves. However, this is a technically demanding procedure that requires prolonged and exhaustive training, with a steep learning curve. tomía. El promedio de edad de los pacientes fue de 15.7 años (12 a 21 años). En relación a los días de internación, se obtuvo un tiempo promedio de permanencia de 9.45 días (5 a 26 días). El promedio entre los valores angulares fue, en el pre operatorio, de $66.25^{\circ}$ Cobb $\left(48^{\circ}\right.$ a $92^{\circ}$ Cobb), y en el postoperatorio fue de $31.2^{\circ} \mathrm{Cobb}\left(15^{\circ}\right.$ a $45^{\circ}$ Cobb). Como complicaciones, cuatro pacientes tenían neuralgia intercostal (20\%) y había un caso de atelectasia (5\%). Conclusiones: la disectomía por videotoracoscopia es un método efectivo y seguro para tornar flexibles las curvas escolióticas rígidas. Sin embargo, se trata de un procedimiento técnicamente dificil, que requiere entrenamiento arduo y prolongado, con curva de aprendizaje extensa.
DESCRITORES: Escoliose; Toracoscopia; Cirurgia torácica vídeo-assistida; Disco intervertebral; Toracotomia
KEYWORDS: Scoliosis;

Thoracoscopy; Thoracic surgery, video-assisted; Intervertebral disk; Thoracotomy

\author{
DESCRIPTORES: Escoliosis; \\ Toracoscopía; Cirugía \\ torácica asistida por video; \\ Disco intervertebral; \\ Toracotomía
}

\section{INTRODUÇÃO}

O tratamento cirúrgico das deformidades da coluna vertebral representa um dos procedimentos mais complexos na prática ortopédica. A escoliose idiopática do adolescente (EIA) é um distúrbio musculoesquelético caracterizado pelo desvio lateral da coluna associado à rotação dos corpos vertebrais, que acomete crianças a partir dos dez anos de idade, e não tem uma causa específica aparente.

Dentro do espectro destas deformidades, é possível encontrar curvas com um componente torácico de maior magnitude ou rigidez, que requerem uma abordagem por via anterior com o objetivo de flexibilizar a curvatura, por meio da discectomia, antes da realização da correção e instrumentação definitivas.

O procedimento padrão para consumar esta liberação é a abordagem anterior por toracotomia ${ }^{1}$. No entanto, esta técnica está associada à morbidade e dor significativas, com complicações que podem incluir comprometimento da função da cintura escapular ${ }^{2}$ e pulmonar $^{3}$.

A cirurgia torácica vídeo-assistida (CTVA) ou videotoracoscopia foi introduzida em 1993 como um método minimamente invasivo de acesso às doenças da coluna vertebral $^{4}$. Desde então, passou a ser utilizada com o intuito de promover a mesma flexibilização proporcionada pela abordagem convencional aberta, mas com a vantagem de reduzir o trauma cirúrgico e melhorar a cosmética do procedimento.
O objetivo deste trabalho é descrever os resultados imediatos com a aplicação desta técnica, tendo como parâmetros o tempo de internação em dias, a porcentagem de correção dos valores angulares no pós-operatório e as complicações inerentes ao ato cirúrgico.

\section{MÉTODOS}

Foram selecionados 20 pacientes, no período de Agosto de 1989 a Maio de 2001, matriculados no Ambulatório de Patologias da Coluna Vertebral do Hospital São Paulo da Escola Paulista de Medicina da Universidade Federal de São Paulo (UNIFESP/EPM), com EIA, e submetidos à cirurgia por dupla abordagem. Inicialmente, realizou-se a abordagem pela via anterior com liberação por videotoracoscopia e depois a via posterior para correção e fixação com instrumental de Hartshill, em um mesmo tempo cirúrgico5.

A finalidade deste estudo foi verificar a aplicabilidade da videotoracoscopia na cirurgia de escoliose, observando como critérios de análise: a correção da curvatura, levando em consideração a porcentagem de correção; o tempo de cirurgia; o tempo de internação; o tempo de permanência em Unidade de Terapia Intensiva (UTI); o uso de dreno torácico; perdas sanguíneas e as complicações inerentes ao ato cirúrgico.

Foram incluídos neste trabalho somente pacientes portadores de escoliose idiopática, padrão de curva torácica direita rígida, King III. O critério utilizado para definir 
uma deformidade como rígida foi correção menor que $40 \%$ da curvatura inicial, após realização da inclinação lateral. Nenhum dos pacientes apresentava doença pulmonar prévia.

\section{RESULTADOS}

Todos os procedimentos com videotoracoscopia foram concluídos com sucesso, sem necessidade de conversão para toracotomia. A média de idade dos pacientes foi de 15,7 anos, variando entre 12 a 21 anos. Em relação aos dias de internação, obteve-se tempo médio de permanência de 9,45 dias, variando de 5 a 26 dias.

A média entre os valores angulares foi, no pré-operatório, de $66,25^{\circ} \mathrm{Cobb}$, variando de $48^{\circ}$ a $92^{\circ} \mathrm{Cobb}$, e no pós-operatório, de $31,2^{\circ} \mathrm{Cobb}$, variando de $15^{\circ}$ a $45^{\circ} \mathrm{Cobb}$.

Toda a abordagem anterior foi realizada pelo mesmo cirurgião, obtendo tempo médio de 98,6 minutos, variando de 57 a 150 minutos, fazendo a discectomia parcial de 4,25 discos intervertebrais em média, variando de três a seis discos, com tempo médio de 10,37 minutos para a discectomia de cada disco intervertebral. A perda sanguínea média foi de 185 mililitros de sangue, variando de 70 a 400 mililitros. No pós-operatório, a drenagem da região torácica foi em média de 3,75 dias, variando de dois a nove dias. Os pacientes de número de ordem 13 e 17 ficaram 9 e 8 dias, respectivamente, com dreno, tendo o paciente de número de ordem 13 apresentado atelectasia pulmonar e, o de número de ordem 17, drenagem sero-sanguinolenta. Os resultados estão apresentados nas Tabelas 1,2 e 3.

\section{DISCUSSÃO}

A utilização de técnicas endoscópicas minimamente invasivas como forma de acesso às doenças da coluna vertebral torácica teve sua aplicação apresentada por Mack et al. na década de $1990^{4}$. A partir de então, houve grande interesse por parte dos cirurgiões, por representar uma forma de abordagem menos agressiva, com dissecções de pele e músculo reduzidas em relação à toracotomia, porém com capacidade semelhante de flexibilização de curvas no tratamento das deformidades rígidas ${ }^{6-9}$. No entanto, trata-se de um procedimento tecnicamente difícil que requer treinamento árduo e prolongado, com curva de aprendizado extensa.

Neste estudo, o tempo cirúrgico médio, contando somente o da via anterior (toracoscopia), foi de 98,6 minutos, variando entre 57 e 150 minutos, o que concorda com o tempo cirúrgico de autores como Holcomb ${ }^{10}$, com 174 minutos, e Rothenberg et al. ${ }^{11}$ com 106 minutos.

A média das curvas dos pacientes no período pré-operatório foi de $66,25^{\circ} \mathrm{Cobb}$, variando entre 48 e $92^{\circ} \mathrm{Cobb}$ e, no pós-operatório, foi para $31,2^{\circ} \mathrm{Cobb}$, com margem entre 15 e $45^{\circ} \mathrm{Cobb}$. A correção média final foi de $52,90 \%$, o que concorda com os dados demonstrados na literatura ${ }^{7,12}$.

Autores como McAfee et al. ${ }^{13}$ Mack et al. ${ }^{14}$ relataram a ocorrência de nevralgia intercostal como complicação transitória, o que também foi observado nos pacientes do presente estudo com números de ordem $9,10,16$ e 18 . A atelectasia pulmonar, diagnosticada em cinco pacientes em cada

TABELA 1 - Apresenta os pacientes submetidos ao estudo, consta o número de ordem, iniciais, mensurações radiográficas do pré-operatório com inclinações e do pós-operatório (em graus)

\begin{tabular}{lcccc}
\hline Número & Iniciais & $\begin{array}{c}\text { Radiografias } \\
\text { pré-operatórias }\end{array}$ & Inclinação & $\begin{array}{c}\text { Radiografias } \\
\text { pós-operatórias }\end{array}$ \\
\hline 1 & FSM & 64 & 55 & 40 \\
2 & ISB & 64 & 52 & 45 \\
3 & CCB & 80 & 62 & 42 \\
4 & EAM & 70 & 51 & 26 \\
5 & JPR & 50 & 45 & 30 \\
6 & LBR & 73 & 48 & 36 \\
7 & CMS & 58 & 43 & 30 \\
8 & JCAS & 58 & 52 & 32 \\
9 & GG & 64 & 42 & 32 \\
10 & TS & 62 & 60 & 27 \\
11 & CCS & 78 & 70 & 33 \\
12 & GCS & 70 & 62 & 39 \\
13 & ME & 92 & 80 & 42 \\
14 & LP & 62 & 50 & 27 \\
15 & AAF & 69 & 40 & 25 \\
16 & RAS & 52 & 48 & 15 \\
17 & RD & 48 & 42 & 16 \\
18 & FP & 61 & 54 & 30 \\
19 & EGS & 80 & 60 & 38 \\
20 & EG & 70 & 60 & 19 \\
\hline
\end{tabular}

Fonte: Dados Extraídos da Tese de Livre Docência do Professor Eduardo Barros Puertas, apresentada na Escola Paulista de Medicina da Universidade Federal de São Paulo, São Paulo, 2001. 
TABELA 2 - Mostra os pacientes com número de ordem, iniciais, tempo cirúrgico da toracoscopia isolada, tempo cirúrgico total (em horas e minutos), dias de internação, tempo de permanência na Unidade de Terapia Intensiva e número de discos retirados

\begin{tabular}{|c|c|c|c|c|c|c|}
\hline Número & Iniciais & $\begin{array}{c}\text { Tempo } \\
\text { cirúrgico da } \\
\text { via anterior }\end{array}$ & $\begin{array}{c}\text { Tempo } \\
\text { cirúrgico total }\end{array}$ & $\begin{array}{c}\text { Dias de } \\
\text { internação }\end{array}$ & $\begin{array}{l}\text { Número de } \\
\text { discos }\end{array}$ & $\begin{array}{c}\text { Tempo na Unidade } \\
\text { de Terapia } \\
\text { Intensiva (dias) }\end{array}$ \\
\hline 1 & FSM & $2 \mathrm{~h} 30 \mathrm{~min}$ & $5 \mathrm{~h} 20 \mathrm{~min}$ & 14 & 3 & \\
\hline 2 & ISB & $1 \mathrm{~h} 30 \mathrm{~min}$ & $4 \mathrm{~h} 35 \mathrm{~min}$ & 9 & 3 & \\
\hline 3 & $\mathrm{CCB}$ & $57 \min$ & $5 \mathrm{~h} 30 \mathrm{~min}$ & 8 & 4 & \\
\hline 4 & EAM & $1 \mathrm{~h} 25 \mathrm{~min}$ & $6 \mathrm{~h}$ & 7 & 3 & \\
\hline 5 & $J P R$ & $1 \mathrm{~h} 45 \mathrm{~min}$ & $5 \mathrm{~h} 50 \mathrm{~min}$ & 7 & 3 & \\
\hline 6 & LBR & $1 \mathrm{~h} 50 \mathrm{~min}$ & $3 \mathrm{~h} 30 \mathrm{~min}$ & 6 & 4 & \\
\hline 7 & CMS & $1 \mathrm{~h} 30 \mathrm{~min}$ & $6 \mathrm{~h} 30 \mathrm{~min}$ & 5 & 4 & \\
\hline 8 & JCAS & $1 \mathrm{~h} 30 \mathrm{~min}$ & $5 \mathrm{~h} 30 \mathrm{~min}$ & 6 & 4 & \\
\hline 9 & GG & $1 \mathrm{~h} 30 \mathrm{~min}$ & $5 \mathrm{~h} 20 \mathrm{~min}$ & 24 & 4 & \\
\hline 10 & TS & $2 \mathrm{~h} 10 \mathrm{~min}$ & $5 h$ & 6 & 5 & \\
\hline 11 & CCS & $1 \mathrm{~h} 20 \mathrm{~min}$ & $6 \mathrm{~h}$ & 5 & 5 & 1 \\
\hline 12 & GCS & $1 \mathrm{~h} 50 \mathrm{~min}$ & $6 \mathrm{~h}$ & 5 & 5 & \\
\hline 13 & $M E$ & $1 \mathrm{~h} 30 \mathrm{~min}$ & $6 \mathrm{~h}$ & 9 & 5 & 1 \\
\hline 14 & $L P$ & $1 \mathrm{~h} 50 \mathrm{~min}$ & $5 \mathrm{~h}$ & 26 & 5 & \\
\hline 15 & AAF & $2 \mathrm{~h}$ & $4 \mathrm{~h} 45 \mathrm{~min}$ & 6 & 4 & \\
\hline 16 & RAS & $1 \mathrm{~h} 45 \mathrm{~min}$ & $4 \mathrm{~h} 45 \mathrm{~min}$ & 14 & 6 & \\
\hline 17 & $\mathrm{RD}$ & $1 \mathrm{~h} 30 \mathrm{~min}$ & $3 \mathrm{~h} 45 \mathrm{~min}$ & 13 & 5 & \\
\hline 18 & $\mathrm{FP}$ & $1 \mathrm{~h}$ & $5 \mathrm{~h}$ & 6 & 4 & \\
\hline 19 & EGS & $2 \mathrm{~h}$ & $4 \mathrm{~h} 45 \mathrm{~min}$ & 7 & 5 & \\
\hline 20 & $E G$ & $1 \mathrm{~h} 30 \mathrm{~min}$ & $6 \mathrm{~h}$ & 6 & 4 & 1 \\
\hline
\end{tabular}

Fonte: Dados Extraídos da Tese de Livre Docência do Professor Eduardo Barros Puertas, apresentada na Escola Paulista de Medicina da Universidade Federal de São Paulo, São Paulo, 2001.

TABELA 3 - Apresenta os pacientes com número de ordem, iniciais, dias com drenagem de tórax, quantidade de perda sanguínea (em mililitros) e complicações

\begin{tabular}{|c|c|c|c|c|c|}
\hline Número & Iniciais & $\begin{array}{l}\text { Complicações da } \\
\text { via anterior }\end{array}$ & $\begin{array}{c}\text { Complicações da via } \\
\text { posterior }\end{array}$ & Dias de dreno & Perda sanguínea \\
\hline 1 & FSM & - & - & 2 & 200 \\
\hline 2 & ISB & - & - & 3 & 220 \\
\hline 3 & $\mathrm{CCB}$ & - & - & 4 & 180 \\
\hline 4 & EAM & - & - & 4 & 100 \\
\hline 5 & JPR & - & - & 2 & 175 \\
\hline 6 & LBR & - & - & 2 & 90 \\
\hline 7 & CMS & - & - & 3 & 70 \\
\hline 8 & JCAS & - & - & 3 & 130 \\
\hline 9 & GG & Nevralgia intercostal & Déficit motor reversível & 3 & 200 \\
\hline 10 & TS & Nevralgia intercostal & Déficit motor reversível & 4 & 300 \\
\hline 11 & CCS & - & - & 3 & 240 \\
\hline 12 & GCS & - & - & 3 & 60 \\
\hline 13 & ME & Atelectasia pulmonar & - & 9 & 200 \\
\hline 14 & LP & - & Déficit motor reversível & 3 & 100 \\
\hline 15 & AAF & - & - & 3 & 125 \\
\hline 16 & RAS & Nevralgia intercostal & Déficit motor reversível & 3 & 400 \\
\hline 17 & $\mathrm{RD}$ & - & - & 8 & 150 \\
\hline 18 & $\mathrm{FP}$ & Nevralgia intercostal & - & 4 & 160 \\
\hline 19 & EGS & - & - & 5 & 300 \\
\hline 20 & EG & - & - & 4 & 300 \\
\hline
\end{tabular}

Fonte: Dados extraídos da Tese de Livre Docência do Professor Eduardo Barros Puertas, apresentada na Escola Paulista de Medicina da Universidade Federal de São Paulo, São Paulo, 2001. 
série destes autores, somente ocorreu em um dos pacientes deste estudo, o de número de ordem 13. Os mesmos autores relataram também a mudança de procedimento de videotoracoscopia para toracotomia, o que não ocorreu nesta série. Em um desses estudos, relatou-se um caso de paraparesia durante a realização da videotoracoscopia ${ }^{14}$, o que não sucedeu com os pacientes deste estudo. Foram obtidos somente quadros neurológicos transitórios nos pacientes com números de ordem 9, 10, 14 e 16, que se reverteram espontaneamente em um curto período de tempo, ocorrendo apenas durante a via posterior e não durante a toracoscopia. Na série de Rosenthal e Dickman ${ }^{15}$, foram apresentadas as complicações da toracotomia, consistindo em $50 \%$ de nevralgia intercostal e $33 \%$ de atelectasia e disfunção pulmonar, contra $16 \%$ de nevralgia intercostal e $7 \%$ de complicações pulmonares relacionadas à toracoscopia. No presente estudo, houve $20 \%$ de nevralgia intercostal (quatro pacientes), com rápida resolução do problema, e 5\% de complicação pulmonar (atelectasia) em um paciente.

O tempo de internação variou de 5 a 26 dias, com tempo médio de 9,45 dias. A permanência dos pacientes internados variou para cada autor e depende evidentemente da doença abordada. Autores como McAfee et al. ${ }^{13}$ tiveram média de internação de 4,97 dias, variando de 2 a 22 dias; Mack et al. ${ }^{14}$, média de 4,82 dias de internação, variando de 2 a 21 dias; Kokoska, Gabriel e Silen, média de 8,6 dias de internação. Arlet, ao fazer uma metanálise de dez artigos com 151 cirurgias, refere uma média de internação de 9,1 dias $^{12}$, dado que, obtido a partir de doenças díspares, não oferece termos de comparação com àqueles levantados na literatura.

$\mathrm{Na}$ presente série, os pacientes com números de ordem 11,13 e 20 ficaram 24 horas internados na UTI. O estudo de Rothenberg et al. relatou permanência média de 1,8 dias $^{11}$, o que corrobora que não há necessidade de cuidados especiais, além dos já tomados na maioria dos pacientes submetidos à toracoscopia e artrodese posterior.

O sangramento médio durante a toracoscopia é de difícil aferição, pois parte do que sangra fica em espaço de difícil acesso e virtualmente impossível de ser aspirado. Nesta série, a média de sangramento foi de aproximadamente $185 \mathrm{~mL}$ (variando de $60 \mathrm{a} 400 \mathrm{~mL}$ ), o que se aproxima dos valores encontrados na literatura, como na metanálise de Arlet, em cujo levantamento obteve-se média de sangramento de $246 \mathrm{~mL}^{12}$.

\section{CONCLUSÃO}

A discectomia por videotoracoscopia é um método efetivo e seguro para tornar flexíveis as curvas escolióticas rígidas. No entanto, trata-se de um procedimento tecnicamente difícil, que requer treinamento árduo e prolongado, com curva de aprendizado extensa.

\section{REFERÊNCIAS}

1. Dwyer AF, Schafer MF. Anterior approach to scoliosis. Results of treatment in fifty-one cases. J Bone Joint Surg Br. 1974;56(2):218-24.

2. Burd TA, Pawelek L, Lenke LG. Upper extremity functional assessment after anterior spinal fusion via thoracotomy for adolescent idiopathic scoliosis: prospective study of twentyfive patients. Spine. 2002;27(1):65-71.

3. Faro FD, Marks MC, Newton PO, Blanke K, Lenke LG. Perioperative changes in pulmonary function after anterior scoliosis instrumentation: thoracoscopic versus open approaches. Spine. 2005;30(9):1058-63.

4. Mack MJ, Regan JJ, Bobechko WP, Acuff TE. Application of thoracoscopy for diseases of the spine. Ann Thorac Surg. 1993;56(3):736-8.

5. Dove J. Luque segmental spinal instrumentation: the use of the Hartshill rectangle. Orthopedics. 1987;10(6):955-61.

6. Bosch R. Thoracoscopic release in scoliosis. Z Orthop Ihre Grenzgeb. 1998;136(2):Oa14-5.
7. Newton PO, Wenger DR, Mubarak SJ, Meyer RS. Anterior release and fusion in pediatric spinal deformity. A comparison of early outcome and cost of thoracoscopic and open thoracotomy approaches. Spine. 1997;22(12):1398-406.

8. Waisman M, Saute M. Thoracoscopic spine release before posterior instrumentation in scoliosis. Clin Orthop Relat Res. 1997;336:130-6.

9. Kokoska ER, Gabriel KR, Silen ML. Minimally invasive anterior spinal exposure and release in children with scoliosis. J Soc Laparoendosc Surg 1998;2(3):255-8.

10.Holcomb GW III, Mencio GA, Green NE. Video-assisted thoracoscopic discectomy and fusion. J Pediatric Surg. 1997;32(7):1120-2.

11. Rothenberg S, Erickson M, Eilert R, Fitzpatrick J, Chang F, Glancy G, et al. Thoracoscopic Anterior Spinal Procedures in Children. J Pediatr Surg. 1998;33(7):1168-71.

12.Arlet V. Anterior thoracoscopic spine release in deformity surgery: a metaanalysis and review. Eur Spine J. 2000;9(Suppl 1):S17-23.
13.McAfee PC, Regan JR, Zdeblick T, Zuckerman J, Picetti GD 3rd, Heim S, et al. The incidence of complications in endoscopic anterior thoracolumbar spinal reconstructive surgery. A prospective multicenter study comprising the first 100 consecutive cases. Spine. 1995;20(14):1624-32.

14.Mack MJ, Regan JJ, McAfee PC, Picetti G, Ben-Yishay A, Acuff TE. Video-assisted thoracic surgery for the anterior approach to the thoracic spine. Ann Thorac Surg. 1995;59(5):1100-6.

15. Rosenthal D, Dickman CA. Thoracoscopic microsurgical excision of herniated thoracic discs. J Neurosurg. 1998;89(2):224-35.

Correspondência:
David Del Curto
Rua Borges Lagoa, 783 - 5 andar
CEP: 04038-031 - São Paulo (SP), Brasil
Tel.:/Fax: (11) 5571-6621
E-mail: ddelcurto@hotmail.com

